\title{
Most important barriers and facilitators of HTA usage in decision- making in Europe
}

\author{
Cheung K.L., Maastricht University \\ Evers S.M.A.A., Maastricht University \\ De Vries H., Maastricht University \\ Levy P., Paris Dauphine University \\ Pokhrel S., Brunel University London \\ Jones T., Brunel University London \\ Danner M., Cologne University Hospital \\ Wentlandt J., Cologne University Hospital \\ Knufinke L., Cologne University Hospital \\ Mayer S, Medical University of Vienna \\ Hiligsmann M, Maastricht University
}




\section{ABSTRACT}

Background: To enhance usage of health technology assessment (HTA) in decision-making, it is important to prioritise important barriers and facilitators to the uptake of HTA. This study aims to quantify and compare the relative importance of barriers and facilitators regarding the use of HTA in several European countries.

Methods: A survey containing two best-worst scaling (BWS) object case studies (i.e. barriers and facilitators) were conducted among 136 policy makers and HTA researchers from the Netherlands, Germany, France, and United Kingdom. Hierarchical Bayes analysis generated the mean relative importance score (RIS) for each factor and subgroup analyses assessed differences between countries.

Findings: Six barriers (RIS $\geq 5$ ) and five facilitators (RIS $\geq 6$ ) were deemed highly important. Eleven barriers and ten facilitators differed in their importance between countries. Policy characteristics, research \& researcher characteristics, and organisation \& resources were particularly important to facilitate uptake of HTA, such as an explicit framework for decision-making and research of sufficient quality.

Discussion: The most paramount barriers and facilitators to HTA usage were quantified. For all countries it is crucial to create an explicit framework for the decision-making context to include HTA evidence. Country differences in the quality of research emphasize the need for enhanced international collaboration in HTA. 


\section{INTRODUCTION}

Due to increasing economic concern, health policy makers need to efficiently allocate resources or select cost-effective health interventions [1]. Health technology assessment (HTA) - a multidisciplinary research field to evaluate the social, economic, organizational and ethical issues of a health intervention (e.g. drugs, medical devices, public health) - aims to facilitate this decision-making process to inform how to obtain value for money [2]. HTA is a policy-oriented form of research designed to inform decision-makers on the relative effectiveness or cost-effectiveness of a new technology compared to current or best practice [3]. Although both the number of public health interventions as well as the number of HTA studies is increasing [4, 5], the uptake of HTA information in the decision-making could be improved $[5,6,7]$.

Due to the relatively young nature of the HTA discipline, scholars do not yet fully understand what may hinder and facilitate the usage of HTA in decision-making and to what extent. Various studies including systematic reviews have been conducted to identify multiple barriers and facilitators to the uptake of HTA information, such as sufficient quality and timeliness of HTA research $[4,5,8,9,10,11$, 12]. However, being aware of the relative importance of each of these factors would provide more guidance to bridge the gap between research and policy. A recent Dutch study identified the most important barriers and facilitators for using HTA in decision-making, using the best-worst scaling (BWS) method [13]. This study suggested that 'policy characteristics', and 'organisation and resources' were particularly important to enhance HTA usage, especially the creation of an explicit framework for the decision-making context including HTA. Despite these valuable insights to the position in the Netherlands, it is important to understand to what extent the relative importance of these factors could be generalizable to other countries, with different decision-making contexts. Countries in Europe and worldwide differ in their level and trends in HTA development [7]. To what extent European countries differ regarding the most important factors and their relative ranking needs yet to be investigated.

Hence, to deepen our understanding of how to enhance HTA uptake in policy making, this study aims to (1) identify the most important barriers and facilitators to HTA usage in the decisionmaking context in four European countries (i.e. Netherlands, Germany, France, and United Kingdom), and (2) explore whether there are country differences and where they differ. 


\section{METHODS}

\section{Best-worst scaling}

In order to elicit expert opinion on the importance and the relative weight of a large list of barriers and facilitators to the use of HTA, we used the BWS method. The BWS method is an increasingly popular conjoint analysis technique [14, 15], devised by Finn and Louviere (1992) [16], having some advantages compared to other methods (such as discrete choice experiments). The BWS method also collects data on the 'worst' option, as well as the 'best' option, resulting in a method capable of incorporating a larger set of factors $[14,17]$, while the object case was shown to be cognitively less burdensome compared to discrete choice experiments for participants [18]. To quantify the relative importance of barriers and facilitators for the use of HTA in policy decisions, this study used the BWS object case. Participants were asked to complete a series of choice tasks in which they had to choose the most and least important factor (i.e. barrier or facilitator) from a list of five factors, derived from a master list.

\section{Participants}

Country-specific researchers (i.e. KLC (Netherlands), JW \& LK (Germany), PL (France), and TJ (United Kingdom)) identified policy makers (i.e. decision makers and advisors on different levels) and HTA experts (i.e. PhD students and senior researchers in HTA) via convenience sampling. Each countryspecific researcher created a list of potential stakeholders in their country based on a search of the relevant health institutes and HTA research departments of several universities, enriched by personal networks. Potential participants were then approached via e-mail, which included a link to the survey (on the Qualtrics platform) which randomly assigned the participant to one of the four versions of the questionnaire for each survey (i.e. barriers and facilitators). A reminder was sent within two weeks to participants who had not completed the survey. All participants were approached between November 2015 and May 2017 (i.e. Netherlands; n=222, Germany; n=365, France; n=109, and United Kingdom; $n=255)$.

\section{Identification of barriers and facilitators}

The master list of factors was drafted based on a scoping review and expert validation in the previous Dutch study [13]. For this, a scoping review using PubMed and Web of Science was conducted to identify important barriers and facilitators regarding the uptake of HTA $[4,5,8,9,10,11,12,19,20$, $21,22,23,24,25,26,27]$. Further details on the search and the selection of attributes can be found in Cheung et al. (2017) [13]. We then defined and validated the list of factors for inclusion in this study. The list of factors was extensively discussed among the working group (i.e. KLC, SE, HdV, and MH), and external experts (two HTA experts and one policy maker) were asked to provide feedback on the list 
regarding (1) completeness, (2) overlap, and (3) wording. This led to a final list of 22 barriers and 19 facilitators for the BWS survey (see Supplementary file 1).

\section{The best-worst scaling survey}

Two surveys (i.e. one for the barriers, one for the facilitators) were designed using the Sawtooth Software's SSI Web platform; resulting in fractional, efficient designs (which are characterised by orthogonality, minimal overlap, positional balance, connectivity, and stability). For each survey four different versions (i.e. different design of the choice-sets) of the questionnaires were used, with 14 choice sets for the barriers and 12 choice sets for the facilitators. The survey consisted of three parts: (1) demographics, (2) barriers, and (3) facilitators; barriers and facilitators were not in a particular order due to randomisation. Qualtrics was used to administer the online survey, and participants were recruited via e-mail. First, participants were asked for their age, gender, and their role (policy maker, HTA expert). Second, Qualtrics then randomised participants to one version of the barriers questionnaire and one version of the facilitators questionnaire. Participants were asked to choose the most important and the least important factor to the uptake of HTA in 14 choice sets for the barriers and 12 choice sets for the facilitators, each consisting of five factors from the master list ( 22 factors for the barriers and 19 factors for the facilitators). In addition, participants were asked to rate the difficulty of the choice tasks using a Likert scale $(0=$ very easy to $7=$ very difficult $)$, for both surveys.

Supplementary files 2 (barriers) and 3 (facilitators) illustrate the BWS choice sets, including the introductory text. The survey was finalised after a pilot with three HTA experts from Maastricht University, and the survey was used in a previous study with the BWS application in the Netherlands [13]. The estimated time to complete the survey is approximately 25 minutes. This survey was then translated to French and German by one or two researchers per country. This version was piloted by five HTA researchers ( $n=2$ for France, $n=3$ for Germany), resulting in minor adjustments without changing the scientific methodology and content.

\section{Analyses}

The analyses were conducted for all completed responses. First, descriptive statistics were calculated to present demographic characteristics of the respondents. Second, to analyse preference data, Hierarchical Bayes analysis $[14,28,29]$ was conducted (using Sawtooth Software's SSI Web platform), which is frequently used to analyse BWS studies in the literature [15]. The mean relative importance score (RIS) with its $95 \%$ confidence interval was calculated for each factor. Rescaled scores were estimated, based on the raw coefficient of the preference function, which represents the probability that the respondent chooses the factor. All factors combined the RIS for each individual sum up to 100 , with a higher score indicating a relatively higher importance of the factor [29]. A RIS of '10' represents 
a two-fold higher importance than a RIS of ' 5 '. Fit statistic was used to identify inconsistent responders. Using the mean RIS, factors were ranked from most to least important for the uptake of HTA in policy making. The individual's fit statistic was used to check the quality of the responses, with a fit statistic lower than 0.25 indicating purely random responses to the choice tasks [30]. In order to check the sensitivity of results, the best-worst count analysis was conducted [20], Last, one-way ANOVA analyses per factor were used for subgroup analyses on 'country' and 'role' to explore whether the perceptions on the importance of these factors differ among different countries and roles, and where the differences lie. Post-hoc analyses were conducted to identify specific country differences, using Tukey honest significant difference (HSD) tests. When homogeneity assumptions were violated, the Welch statistic was used to identify main effects, while using Games-Howell tests for the post-hoc analyses.

\section{RESULTS}

\section{Descriptive statistics}

In total, 298 stakeholders started the survey, of which 136 (45.8\%) actually completed the survey. Of the 162 incomplete responses, 129 responses (80\%) contained complete information on the demographic characteristics (i.e. gender, age, and role). Participants who completed the survey did not significantly differ from participants who dropped out, except for in terms of their 'role' (defined as either policy maker or HTA researcher). Policy makers dropped out significantly more than HTA researchers $\left(\chi^{2}(1, N=265)=6.17, p=0.01\right)$. Stakeholders rated both BWS experiments as moderately difficult on a 7-point Likert scale (barriers, Mean=4.54, SD=1.38; facilitators, Mean=4.54, SD=1.38). Table 1 provides information on the demographic characteristics (i.e. gender, age, and role).

\section{Relative importance of the barriers}

The RIS scores of the barriers, overall and for each country, are depicted in Table 2. First, we identified the overall most important barriers to HTA usage in the decision-making context. Overall, a cut-off RIS score of 5 shows six highly important barriers: (1) No explicit framework for decision making process (that uses HTA evidence), (2) Limited generalizability (of HTA studies to the policy maker's context), (3) No availability of relevant HTA research (for policy makers), (4) Lack of consensus between HTA findings (existence of different and opposing recommendations), (5) Insufficient quality (of HTA research findings: quality not according scientific requirements), and (6) No access to relevant HTA research (or poor dissemination) for policy makers.

Second, we explored the potential country differences. Subgroup analyses revealed that 11 barriers differed in their importance between countries: (1) Lack of timeliness of research, (2) Lack of longstanding relation between professionals/policymakers and researchers, (3) Lack of contact and 
interaction, among policy-makers, HTA researchers, and other stakeholders, (4) No availability of relevant HTA research for policy makers, (5) No access to relevant HTA research (or poor dissemination) for policy makers, (6) Absence of policy networks, (7) Lack of support, within the organization to the use of HTA, (8) Insufficient quality, of HTA research findings: quality not according scientific requirements, (9) Policy-maker's perception of insufficient support by end-users (e.g. patient associations), (10) Lack of legal or legislative support to the use of HTA, and (11) Absence of appropriate incentives, for implementation of cost-effective interventions. Remarkable country differences were 'Insufficient support by stakeholders' (i.e. Netherlands with a noticeably higher RIS scores than the other countries) and 'No availability of relevant HTA research' (i.e. Netherlands with a noticeably lower RIS scores than the other countries).

For policy makers specifically, subgroup analyses showed seven significant differences in RIS scores between countries for policy makers (see Figure 1): (1) Lack of longstanding relation (NL,DE>FR), (2) Lack of contact and interaction (NL>FR), (3) No availability of relevant HTA research (DE,FR>NL), (4) Absence of policy networks (NL,DE>FR), (5) Insufficient support by stakeholders (NL>DE,FR,UK), (6) Insufficient quality, of HTA research findings (DE,FR>NL), and (7) Insufficient legal support (NL>DE,FR,UK). The best-worst count analysis revealed a similar ranking (slight differences) (results available upon requests from the corresponding author). Supplementary file 4 details additional analyses.

\section{Relative importance of the facilitators}

The RIS scores of the facilitators, overall and for each country, are depicted in Table 3. First, the overall most important facilitators to HTA usage in the decision-making context were identified. When a cutoff RIS score of 6 is used (which is different from the barriers due to fewer factors resulting into higher RIS scores per factor), overall five facilitators were deemed highly important by the participants: (1) Sufficient quality, (2) Availability of explicit framework for decision making process, (3) Availability to relevant HTA research, (4) Sufficient qualified human resources, and (5) Higher transparency of HTA research findings.

Second, potential country differences were investigated. Subgroup analyses showed that ten facilitators significantly differed in their importance between countries; (1) Improving longstanding relation, (2) Sufficient legal support, (3) More personal contact and interaction, (4) Sufficient support by stakeholders, (5) Creation of policy networks, (6) Availability of guidelines, (7) Appropriate incentives, (8) Access to relevant HTA research, (9) Availability to relevant HTA research, and (10) Sufficient quality. In line with the barriers, remarkable country differences were 'Availability of relevant HTA research' and 'Sufficient support by stakeholders'. 
Regarding policy makers only, subgroup analyses revealed seven significant differences in RIS scores between countries, which were: (1) 'Sufficient quality' (DE,FR>NL), (2) 'Sufficient credibility' (DE>NL), (3) 'Improving longstanding relation' (NL,DE>FR), (4) 'Creation of policy networks' (NL>FR), (5) 'More personal contact and interaction' (NL,DE>FR), (6) 'Sufficient support by stakeholders' (NL>DE,UK,FR), and (7) 'Availability of guidelines' (FR>NL,DE) (see Figure 2). The best-worst count analysis [20] revealed a similar ranking (slight differences) (results available upon requests from the corresponding author).

\section{DISCUSSION}

This study aimed to identify the most important barriers and facilitators to HTA usage in the decisionmaking context in four European countries and to explore potential country differences. Overall, the most important barrier is the lack of an explicit framework for the decision making process (that uses HTA evidence). At the same time, the availability of an explicit framework for the decision making process (that uses HTA evidence) is among the most important facilitators. This is in line with findings from García-Mochón et al. (2017) [31], suggesting that a prerequisite for the adoption of HTA evidence is a transparent and comprehensive system, and therefore of utmost importance to facilitate its usage in decision-making.

Based on the most important barriers, it seems that - as categorised by Oliver et al. (2014) [5] - policy characteristics (e.g. 'No explicit framework'), research and researcher characteristics (e.g. 'Limited generalizability', 'Lack of consensus between HTA findings', 'No availability of relevant HTA research', and 'Insufficient quality'), and organisation and resources (e.g. 'No access to relevant HTA research') are important to tackle to enhance HTA usage. Next to the availability of an explicit framework, the most important facilitators overall were: 'Sufficient quality', 'Availability to relevant HTA research', 'Sufficient qualified human resources', and 'Higher transparency of HTA research findings'; again pointing at the importance of addressing policy characteristics, research and researcher characteristics, and organisation and resources, as found for barriers and as categorised by Oliver et al. (2014) [5]. Similarly to the previous study in the Netherlands [13], many of the most important barriers paralleled the facilitators, meaning that the most important facilitators were the factors that targeted the most important barriers. For instance the barrier 'Insufficient quality' (of HTA studies), and the facilitator 'Sufficient quality' (of HTA studies) were both among the most important factors. Findings indicate a lack of qualified human resources to conduct or understand relevant HTA research within the policy organization, suggesting the need to improve capability in health economics by employing more health economists and providing further training for policy makers. It seems that 
over the years, the quality of HTA studies and lack of qualified people have remained important issues to address. This finding is consistent with a study from 2010, indicating the need for more training in health economics, and the lack of quality [32].

This study further identified the country differences regarding the relative importance of the barriers and facilitators. Differences were found in several barriers and facilitators, such as in the importance of the availability of relevant HTA research. The Netherlands markedly differ from the other countries where the availability of relevant HTA is among the most important facilitators. In addition, overall the quality of HTA studies is deemed the most important facilitator. All countries seem to agree that it is both, an important barrier and facilitator, yet Dutch participants prioritised sufficient quality significantly lower than the German participants. For instance, compared to Germany, Dutch participants perceived having appropriate incentives for implementation of cost-effective interventions, and the availability of an explicit framework for decision making process more important than sufficient quality of HTA research. Countries generally differ in their level and trends in HTA development (e.g. the UK having an advanced HTA program) [7]. Perhaps the country difference regarding the (lack of) sufficient quality is due to differences in HTA development between these countries, with for example the Netherlands having a long tradition in establishing a comprehensive HTA program. Overall, the high importance and heterogeneity of 'insufficient quality of HTA research' and 'availability of relevant HTA research' indicate the need for enhanced international collaboration in HTA. Due to sharing experience, skills, and methodology, international collaboration is an efficient way to increase e.g. the variety of high-quality HTA information and the number of timely national HTA reports $[3,22,33,34]$. In a study on the collaboration in HTA (EUnetHTA joint action 2010-2012), facilitating factors were suggested to enhance international collaboration, e.g. merging methodological and clinical expertise, and acceptance of English-written reports by decision makers [22]. As indicated in a study from 2000, credibility of HTA studies is an important factor to enhance usage of HTA [32]. Yet, this was not reflected in our findings, which may indicate a general shift e.g. due to increased transparency standards in the past 15 years $[35,36]$.

Furthermore, HTA policy assumes that the incorporation of technologies in health systems could be improved by scientific rationality and unite the different stakeholders around the same objectives. Overall, our findings indicate that HTA policy depends on a virtuous combination between a science policy (e.g. good quality and adequate studies), and a health care policy (e.g. adequate decision-making framework). All barriers and facilitators seem related to one of these two poles. Hence, further research may shed light to validate these pools and which barriers and facilitators of each pole are the most important. Next to that, further research could investigate how these factors relate to important individual beliefs that guide behaviour, which may lead to an enhanced 
understanding of these factors in a socio-cognitive context (e.g. placing the barriers and facilitators under the I-Change model) [37, 38].

This study has strength and limitations. This study is first to use conjoint analysis to quantify importance of barriers and facilitators to HTA usage in decision-making. Findings could serve as a first step to prioritise the identified barriers and facilitators from literature. One limitation may be that the master lists of the barriers and facilitators were not validated specifically for all four included countries [39]. Country-specific factors that potentially could be highly important may thus have been neglected, while some factors may not be relevant in the respective country. Qualitative research for each country could aid the findings, providing insights which factors are relevant for the respective country and why. However, to ensure comparability between cross-country findings, it was important for the lists of barriers and facilitators to be identical in this BWS study. Moreover, the identified factors were based on a scoping review of international literature, and were validated among British and Dutch experts. Another limitation regards translations; while the translation of the survey was conducted by one or two researchers per country, no back-translation was carried out to check its accuracy. Therefore, minor differences in the translations may have impaired the comparability of the survey across countries. Finally, due to convenience sampling, the generalisability may be limited. Policy makers were also found to be more likely to opt out of the survey than HTA experts. More HTA experts than policy makers were eventually included in the survey, implying that the average scores presented in this study are better capturing the perception of HTA experts. Hence, looking at the two groups of stakeholders separately as presented in Figure 1 and 2 is vital to get a more nuanced picture of policy makers specifically. An opportunity for future research regards the generalisability of findings to outof-sample countries, especially in emerging countries with a different decision-making context [22, 32]. Middle-income countries (e.g. India and Russia) compared to high-income countries (e.g. Netherlands and United Kingdom), have less developed HTA programs although the community that uses HTA is growing in middle-income countries as well [7]. Our findings may therefore not be fully transferable to other countries, particularly to middle-income countries. At the same time, this study included different healthcare systems (e.g. national health care system like the United Kingdom and social security system like Germany) in Western Europe. This study may be a stepping stone to draw further comparisons with less wealthy European countries and beyond Europe.

\section{CONCLUSIONS}

This study revealed the most important barriers and facilitators to HTA usage in the decision-making context, and differences in four European countries (i.e. Netherlands, Germany, France, and the UK) 
were explored. Policy characteristics, research and researcher characteristics, and organisation and resources were found to be particularly important to address in order to facilitate uptake of HTA. It is also crucial to create a national impulse to make a political statement and create an explicit framework for the decision-making context to include HTA evidence. In addition, it seems that generating high quality HTA research in the first place is a prerequisite for adoption. Heterogeneity in the quality of research point out the need for enhanced international collaboration in HTA, which in turn may increase e.g. high-quality HTA information and the number of timely national HTA reports.

\section{KEY ISSUES}

- Policy characteristics, research and researcher characteristics, and organisation and resources were found to be particularly important to facilitate uptake of HTA in the decision-making context, such as an explicit framework for decision-making and improving quality of HTA research.

- Findings indicate a lack of qualified human resources to conduct or understand relevant HTA research within the policy organization, suggesting the need to improve capability in health economics by employing more health economists and providing further training for policy makers.

- The most important factor seems to be an explicit framework for the decision-making context to include HTA evidence.

- Generating high quality HTA research seems to be a prerequisite for adoption. Heterogeneity in the relative importance scores regarding the quality of research point out the need for enhanced international collaboration in HTA, which in turn may increase e.g. high-quality HTA information and the number of timely national HTA reports. 


\section{REFERENCES}

1. Park AL, McDaid D, Weiser P, et al. Examining the cost effectiveness of interventions to promote the physical health of people with mental health problems: a systematic review. BMC public health. 2013;13:787. doi: 10.1186/1471-2458-13-787. PubMed PMID: 23988266; PubMed Central PMCID: PMC3765875.

2. Nicod E, Kanavos P. Commonalities and differences in HTA outcomes: a comparative analysis of five countries and implications for coverage decisions. Health policy. 2012;108(2):167-177.

3. Drummond MF, Schwartz JS, Jönsson B, et al. Key principles for the improved conduct of health technology assessments for resource allocation decisions. International journal of technology assessment in health care. 2008;24(03):244-258.

4. van Velden ME, Severens JL, Novak A. Economic evaluations of healthcare programmes and decision making. Pharmacoeconomics. 2005;23(11):1075-1082.

5. Oliver K, Innvar S, Lorenc T, et al. A systematic review of barriers to and facilitators of the use of evidence by policymakers. BMC health services research. 2014;14:2 doi: 10.1186/14726963-14-2. PubMed PMID: 24383766; PubMed Central PMCID: PMC3909454.

6. Macintyre $S$, Chalmers I, Horton R, et al. Using evidence to inform health policy: case study. BMJ: British Medical Journal. 2001;322(7280):222.

7. Oortwijn W, Broos $\mathrm{P}$, Vondeling $\mathrm{H}$, et al. Mapping of health technology assessment in selected countries. International journal of technology assessment in health care. 2013;29(04):424-434.

8. Garrido MV. Health technology assessment and health policy-making in Europe: current status, challenges and potential. WHO Regional Office Europe; 2008. (14).

9. Drummond M. Economic evaluation in health care: Is it really useful or are we just kidding ourselves? Australian Economic Review. 2004;37(1):3-11.

10. Neumann PJ. Why don't Americans use cost-effectiveness analysis. Am J Manag Care. 2004;10(5):308-312.

11. Neumann PJ, Sullivan SD. Economic Evaluation in the US. Pharmacoeconomics. 2006;24(11):1163-1168.

12. Prosser LA, Koplan JP, Neumann PJ, et al. Barriers to using cost-effectiveness analysis in managed care decision making. The American journal of managed care. 2000;6(2):173-179.

13. Cheung KLE, Silvia Vries, Hein Hiligsmann, Mickael. Most important barriers and facilitators regarding the use of Health Technology Assessment. International Journal of Technology Assessment in Health Care. 2017;33(2):1-9.

14. Mühlbacher AC, Kaczynski A, Zweifel P, et al. Experimental measurement of preferences in health and healthcare using best-worst scaling: an overview. Health economics review. 2015;6(1):1-14.

15. Cheung KL, Wijnen BF, Hollin IL, et al. Using Best-Worst Scaling to Investigate Preferences in Health Care. PharmacoEconomics. 2016;34(12):1195-1209.

16. Finn A, Louviere JJ. Determining the appropriate response to evidence of public concern: the case of food safety. Journal of Public Policy \& Marketing. 1992:12-25.

17. Flynn TN, Louviere JJ, Peters TJ, et al. Best-worst scaling: what it can do for health care research and how to do it. Journal of health economics. 2007;26(1):171-189.

18. Marley AA, Louviere JJ. Some probabilistic models of best, worst, and best-worst choices. Journal of Mathematical Psychology. 2005;49(6):464-480.

19. Merlo G, Page K, Ratcliffe J, et al. Bridging the gap: exploring the barriers to using economic evidence in healthcare decision making and strategies for improving uptake. Applied health economics and health policy. 2014:1-7.

20. van Gool MK, Gallego G, Haas M, et al. Economic Evidence at the Local Level. Pharmacoeconomics. 2007;25(12):1055-1062. 
21. Williams I, Bryan S. Understanding the limited impact of economic evaluation in health care resource allocation: a conceptual framework. Health policy. 2007;80(1):135-143.

22. Huić $M$, Nachtnebel A, Zechmeister I, et al. COLLABORATION IN HEALTH TECHNOLOGY ASSESSMENT (EU net HTA JOINT ACTION, 2010-2012): FOUR CASE STUDIES. International journal of technology assessment in health care. 2013;29(03):323-330.

23. Drummond $\mathrm{M}$, Weatherly $\mathrm{H}$. Implementing the findings of health technology assessments. International Journal of Technology Assessment in Health Care. 2000;16(01):1-12.

24. Hivon $\mathrm{M}$, Lehoux $\mathrm{P}$, Denis J-L, et al. Use of health technology assessment in decision making: Coresponsibility of users and producers? International journal of technology assessment in health care. 2005;21(02):268-275.

25. STAKES $H$, Rius ME, Espinas JA. EUR-ASSESS project subgroup report on dissemination and impact. International Journal of Technology Assessment in Health Care. 1997;13(2):220-286.

26. Brousselle A, Lessard C. Economic evaluation to inform health care decision-making: promise, pitfalls and a proposal for an alternative path. Social science \& medicine. 2011;72(6):832839.

27. Hoffmann C, Stoykova BA, Nixon J, et al. Do health-care decision makers find economic evaluations useful? The findings of focus group research in UK health authorities. Value in Health. 2002;5(2):71-78.

28. Johnson RM. Understanding HB: an intuitive approach. Sawtooth Software Inc, Sequim, WA. 2000.

29. Orme B. Hierarchical Bayes: Why all the attention? Quirk's Marketing Research Review. 2000;14(3):16-63.

30. Sawtooth Software. Identifying 'bad' respondents: Fit Statistic and Identifying Random Responders. 2016 [cited 2016 January 27th ]; Available from:

https://www.sawtoothsoftware.com/help/issues/ssiweb/online help/hid web maxdiff bad respondents.htm.

31. García-Mochón L, Balbino JE, de Labry Lima AO, et al. HTA and decision-making processes in Central, Eastern and South Eastern Europe: Results from a survey. Health Policy. 2017.

32. Hoffmann C, von der Schulenburg J-MG. The influence of economic evaluation studies on decision making.: A European survey. Health policy. 2000;52(3):179-192.

33. Kristensen FB. Development of European HTA: from vision to EUnetHTA. Michael. 2012;9:147-156.

34. Mäkelä M, Rosén $M$, Jamtvedt $G$, et al. Cooperation is strength: Joint achievements of the Nordic HTA centers. Michael. 2012;9:137-146.

35. Drummond MF, Schwartz JS, Jonsson B, et al. Key principles for the improved conduct of health technology assessments for resource allocation decisions. Int J Technol Assess Health Care. 2008 Summer;24(3):244-58; discussion 362-8. doi: 10.1017/s0266462308080343. PubMed PMID: 18601792; eng.

36. Stephens JM, Handke B, Doshi JA. International survey of methods used in health technology assessment (HTA): does practice meet the principles proposed for good research. Comp Eff Res. 2012;2:29-44.

37. Cheung KL, Evers SM, Hiligsmann M, et al. Understanding the stakeholders' intention to use economic decision-support tools: A cross-sectional study with the tobacco return on investment tool. Health Policy. 2016 Jan;120(1):46-54. doi: 10.1016/j.healthpol.2015.11.004. PubMed PMID: 26718686; eng.

38. De Vries H. An integrated approach for understanding health behavior; the I-Change Model as an example. Psychology and Behavioral Science International Journal. 2017;2(2):555-585.

39. Feig $\mathrm{C}$, Cheung KL, Hiligsmann $\mathrm{M}$, et al. Best-worst scaling to assess the most important barriers and facilitators for the use of health technology assessment in Austria. Expert review of pharmacoeconomics \& outcomes research. 2017 Sep 8:1-10. doi: 10.1080/14737167.2017.1375407. PubMed PMID: 28862060; eng. 
